\title{
Is the Prefrontal Cortex Necessary for Establishing Cognitive Sets?
}

\author{
James B. Rowe, ${ }^{1,2,3}$ Katsuyuki Sakai, ${ }^{4}$ Torben E. Lund, ${ }^{3,5}$ Thomas Ramsøy, ${ }^{3}$ Mark Schram Christensen, ${ }^{3,6}$ \\ William F. C. Baare, ${ }^{3,7}$ Olaf B. Paulson, ${ }^{3,8,9}$ and Richard E. Passingham ${ }^{10,11}$ \\ ${ }^{1}$ Department of Clinical Neurosciences, Cambridge University, Cambridge CB2 2QQ, United Kingdom, ${ }^{2}$ Medical Research Council Cognition and Brain \\ Sciences Unit, Cambridge CB2 7EF, United Kingdom, ${ }^{3}$ Danish Research Centre for Magnetic Resonance, DK-2650 Copenhagen, Denmark, ${ }^{4}$ Department of \\ Cognitive Neuroscience, University of Tokyo, Bunkyo-ku, Tokyo 113-0033, Japan, ${ }^{5}$ Danish National Research Foundation's Centre for Functionally \\ Integrative Neuroscience, Aarhus University, 8000 Aarhus, Denmark, ${ }^{6}$ Department of Exercise and Sport Sciences, University of Copenhagen, DK-2200 \\ Copenhagen, Denmark, ${ }^{7}$ Centre for Integrated Molecular Brain Imaging, DK-2100 Copenhagen, Denmark, ${ }^{8}$ Neurocentre, Rigshospitalet, DK-2100 \\ Copenhagen, Denmark, ${ }^{9}$ Neurobiology Research Unit, DK-2100 Copenhagen, Denmark, ${ }^{10}$ Wellcome Trust Centre for Neuroimaging, London WC1N 3BG, \\ United Kingdom, and ${ }^{11}$ Department of Experimental Psychology, University of Oxford, Oxford OX1 4AU, United Kingdom
}

There is evidence from neuroimaging that the prefrontal cortex may be involved in establishing task set activity in advance of presentation of the task itself. To find out whether it plays an essential role, we examined patients with unilateral lesions of the rostral prefrontal cortex. They were first instructed as to whether to perform a spatial or a verbal working memory task and then given spatial and verbal items after a delay of 4-12 s. The patients showed an increase in switch costs, making more errors by repeating what they had done on the previous trial. They were able to establish regional task set activity during the instruction delay, as evidenced by sustained changes in the blood oxygenation level-dependent signal in caudal frontal regions. However, in contrast to healthy controls, they were less able to maintain functional connectivity among the surviving task-related brain regions, as evidenced by reduced correlations between them during instruction delays. The results suggest that the left rostral prefrontal cortex is indeed required for establishing a cognitive set but that the essential function is to support the functional connectivity among the task-related regions.

Key words: prefrontal cortex; fMRI; connectivity; lesion; cognitive set; switch

\section{Introduction}

In everyday life, we can anticipate tasks or events and prepare the appropriate mental processes or responses. These processes are known as "sets," reflecting preparation for tasks to be performed in the immediate future. They are important in the regulation of behavior and are closely associated with the functions of the lateral prefrontal cortex (Miller and Cohen, 2001), including the maintenance of a specified set (MacDonald et al., 2000; Sakai and Passingham, 2003, 2006) and switching between sets (Rogers et al., 2000; Rushworth et al., 2002, 2005). Set-shifting deficits are also observed with lesions of prefrontal cortex (Owen et al., 1993; Dias et al., 1997; Manes et al., 2002; Aron et al., 2004).

Event-related functional magnetic resonance imaging (fMRI) can be used to distinguish the neural correlates of set from the other task components (Toni et al., 1999). In this way, one can identify rostral prefrontal activations related to multiple cognitive sets, which are temporally distinct from performance of the predicted task itself and spatially distinct from the task-specific,

Received May 23, 2007; revised Aug. 17, 2007; accepted Sept. 13, 2007.

This work was supported by the Wellcome Trust, United Kingdom (J.B.R., K.S., R.E.P.); the University of Copenhagen (J.B.R., T.E.L., M.S.C., W.F.C.B., O.B.P.); and the Simon Spies Foundation, Denmark.

Correspondence should be addressed to Dr. James B. Rowe, Medical Research Council-Cambridge University, 15 Chaucer Road, Cambridge CB2 2QQ, UK. E-mail: james.rowe@mrc-cbu.cam.ac.uk. DOI:10.1523/JNEUROSCI.2349-07.2007

Copyright $\odot 2007$ Society for Neuroscience 0270-6474/07/2713303-08\$15.00/0 set-related activations in nonprefrontal cortex (Sakai and Passingham, 2003, 2006). This rostral prefrontal activity occurs regardless of the semantic, phonological, or visuospatial nature of the preconfigured task. It has therefore been proposed that the prefrontal cortex is the source of the preconfiguration of appropriate cognitive processes, where these processes themselves are instantiated in caudal brain regions.

This is indirectly supported by the behavioral deficits after damage to rostral prefrontal cortex. Patients with frontal lesions may not use advanced information to prepare motor responses (Koski et al., 1998; Lepage and Richer, 2000), make predictive judgments (Gomez-Beldarrain et al., 2004) or engage in appropriate anticipatory behavior (Karnath et al., 1991), or change behavioral rules even when the change of rule is given or predicted (Stablum et al., 1994; Rogers and Monsell, 1995; Rogers et al., 1998; Warrington, 2000).

We used the design of Sakai and Passingham $(2003,2006)$ to study the effects of rostral prefrontal lesions in humans on the prediction and performance of two working memory tasks. We studied four patients with left unilateral lesions. We chose left lesions because of evidence that impairment of maintenance of rules is more associated with left frontal lesions (Goldberg et al., 1994; Burgess et al., 2000). We expected that the patients would perform the forward span tasks normally because unilateral left or right prefrontal lesions have minimal impact on such tasks 
(Owen et al., 1996; D’Esposito and Postle, 1999) and that during the task itself, there would be normal memory-related activity in the intact modality-specific regions.

However, we predicted that the patients would be unable to activate the regions that are specific to each modality, in advance of the presentation of the memory items because of an inability to establish the appropriate cognitive set. We also predicted that the patients would have impaired functional connectivity among the modality specificregions. This was based on previous observations that activity in the rostral prefrontal cortex predicted the correlation between area 8 and parietal cortex during a spatial working memory task (Sakai et al., 2002).

\section{Materials and Methods}

Participants. Nineteen healthy adult subjects [nine men; age, 21-61 years (mean, 41; SD , 12)] were recruited from a departmental register of volunteers in Copenhagen. None had a history of significant neurological, psychiatric, or cardiac disease, and none took regular medication.

Patients with lesions of the prefrontal lobe were recruited through the Copenhagen University Hospital. The clinical details are summarized in Table 1. The principal indication for inclusion was a unilateral tumor of the left rostral prefrontal cortex, sparing Broca's area and the premotor cortex. Apart from previous tumor-associated seizures, none had a history of other significant neurological, cardiac, or psychiatric history. We sought young patients ( $<65$ years) with nonmetastatic lesions, to reduce the likelihood of diffuse ischemic or paraneoplastic changes in the brain. All patients were competent to give consent. The study was approved by the Copenhagen district research ethics committee.

Behavioral paradigm. The principal working memory paradigm was adapted from Sakai and Passingham (2003), using the forward memory span of four sequential letters or four sequential spatial locations, as shown in Figure 1. On each trial, subjects were first presented with an instruction as to whether to remember the spatial or verbal items. There followed an instruction delay of between 4 and $12 \mathrm{~s}$ in steps of $2 \mathrm{~s}$. This was followed by the presentation of the stimuli to be remembered. Four images were shown in rapid succession (600 ms display, $200 \mathrm{~ms}$ interval), each with a capital letter and a separate red square. In each image, the letter and location were in separate quadrants. The sequence of letters or locations was remembered during a second "memory" task period lasting $6 \mathrm{~s}$, until a probe stimulus questioned during $4 \mathrm{~s}$ whether one specific letter or location was followed immediately by another specific letter or location in the sequence. The participants pressed the index finger for "yes" and the middle finger for "no" (or vice versa in half of the subjects). There was an intertrial interval of $8 \mathrm{~s}$.

The participants were pretrained outside the scanner with two sets of 30 trials on the same day, using a different set of stimuli. During scanning, they performed 44 trials. On half of these trials, the instruction was to remember the locations and half the letters. The order of trials was permuted, so that no more than three trials of any one type would be presented in a row, and there were an equal number of trials in which the modality switched or was repeated.

The stimuli were presented using E-prime (Psychology Software Tools, Pittsburgh, PA) and IFIS-SA System (MRI Devices, Waukesha, WI) software, in Windows 98 environment (Microsoft, Redmond, WA). The response accuracy and reaction time to the probe stimulus were recorded from each trial. Group effects were assessed by three-way repeated-measures ANOVA (SPSS 11.0; SPSS, Chicago, IL) with stimulus modality (verbal vs spatial) and switch (switch vs stay) as withinsubject factors and group (control vs patient) as the between-subject factor.

The participants also underwent a psychological evaluation with the Danish Adult Reading Test (DART), analogous to the NART (National Adult Reading Test). This correlates highly with the Weschler Adult

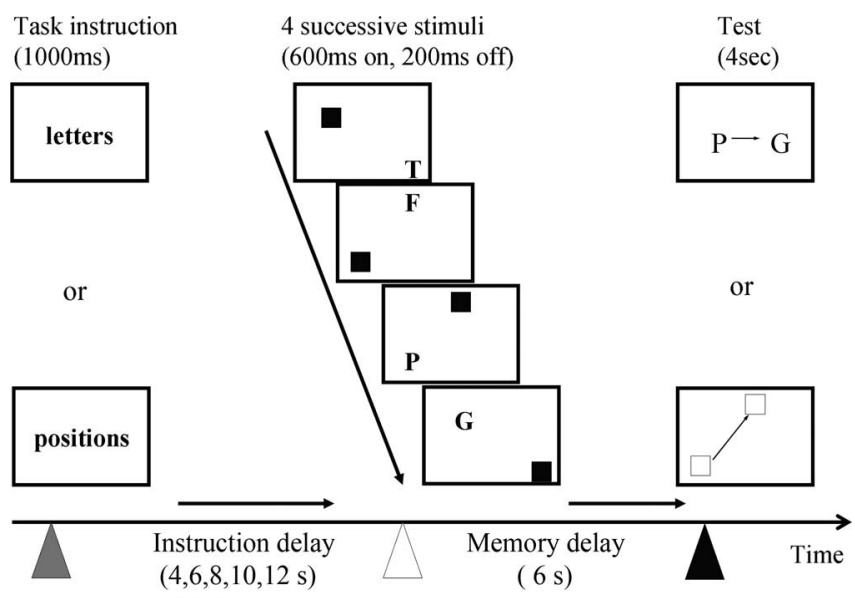

Figure 1. The task gave subjects advanced warning of the modality of a forthcoming verbal (letter-based) or spatial (position-based) working memory task allowing the subjects to establish the relevant cognitive set in advance of the actual stimuli. This "instruction delay" varied between 4 and $12 \mathrm{~s}$. The stimuli each included a letter and square of specific location, but only one modality needed to be attended to and remembered. The order of letters or locations was remembered for $6 \mathrm{~s}$ and tested by a single probe cue that asked about the order of two of the four remembered stimuli (e.g., in these examples, was the letter $P$ followed immediately by the letter $\mathrm{G}$, or was the bottom left square followed immediately by the top square?) (yes to both examples).

Intelligence Scale WAIS-R VIQ, and is resistant to frontal lobe damage. Thus, they can be used as estimators of the premorbid intelligence quotient (Blair and Spreen, 1989; Spreen and Strauss, 1991)

Imaging data acquisition and preprocessing. Functional magnetic resonance images were acquired with $\mathrm{T} 2 *$ blood oxygenation leveldependent (BOLD)-sensitive echo-planar imaging (EPI) [repetition time (TR), $1.78 \mathrm{~ms}$; echo time (TE), $30 \mathrm{~ms}$; flip angle (FA), 82] in $30 \times 4$ $\mathrm{mm}$ interleaved slices $(64 \times 64$ voxels; in-plane resolution, $3 \mathrm{~mm})$ on a Siemens (New York, NY) Trio scanner operating at 3 Tesla at the Danish Research Centre for Magnetic Resonance, Copenhagen University Hospital (Hvidovre, Denmark). Seven hundred fifty images were acquired during $22 \mathrm{~min}$ continuous scanning. T1-weighted, high-resolution magnetization-prepared rapid gradient echo (MPRAGE) images (TR, $1540 \mathrm{~ms}$; TE, $3.93 \mathrm{~ms}$; inversion time, $800 \mathrm{~ms}$; FA, 9; isotropic $1 \mathrm{~mm}$ voxels) were acquired on all subjects to facilitate anatomic localization and determine lesion size.

The preprocessing of images used SPM5 (www.fil.ion.ucl.ac.uk/spm). Images were sinc interpolated in time to correct for phase differences during acquisition and realigned to the first image. The mean functional image was coregistered to the MPRAGE. The MPRAGE was transformed to normal anatomic space (Talairach and Tournoux, 1988) based on the Montreal Neurological Institute (MNI) mean brain by linear and smoothly nonlinear transformations (Friston et al., 1995), and the normalization parameters werer applied to the functional, mean, and structural images. For patients, the mean EPI image was masked during normalization (weight set to zero) (Rorden and Brett, 2000) with a mask that removed areas of lesion and a $\sim 1 \mathrm{~cm}$ margin. Masks were created using MRIcro software (www.psychology.nottingham.ac.uk/ /mricro.html). The data were then smoothed spatially with a Gaussian kernel of $10 \mathrm{~mm}$ 


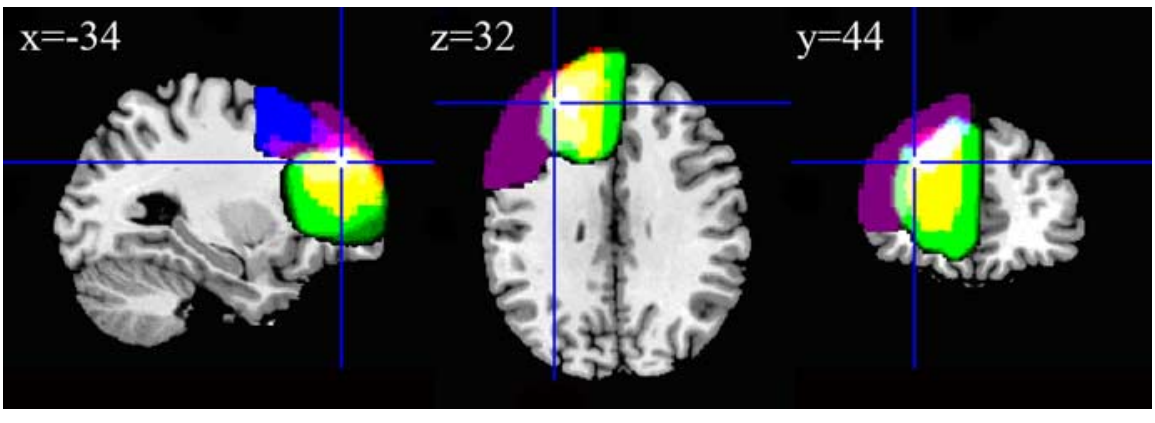

Figure 2. The four patients' (P1-P4) lesions are shown overlaid on the canonical brain in MNI space. The four patients are separately color coded: P1, purple, overlapping as pink, white); P2, blue, overlapping as light pink and light purple, white); P3, green, overlapping as yellow, white); P4, red, overlapping as yellow, white). Complete overlap of all lesions is indicated by white. The cross-hairs are placed at the peak of prefrontal activation associated with set-related activity in previous studies (Sakai et al., 2006).

at full-width half-maximum. In addition, lesions were drawn manually using MRIcro software on the MPRAGE sequence.

Imaging data analysis. The fMRI data were analyzed using general linear models within SPM5, following the general model structure for each subject used by Sakai et al. $(2003,2006)$. In the first level model for each subject, epoch-like covariates were included that specified sustained activation during the instruction delay (4-12 s) for verbal and spatial tasks separately and during the memory delay from the onset of stimuli ( 9 s) for each task modality. Transient responses were included for the events of task instruction (common to all trials) and a "miniblock" for the response to the probe (3s) for each domain. Correct trials were modeled conjointly within each modality. Each error trial was modeled separately, not conjointly. All task covariates were convolved by the canonical hemodynamic response function.

Between-subject effects were modeled at the second level. For each contrast of interest at the first level, a contrast image was taken forward to a second-level model. These second-level models were of three types: (1) one-sample $t$ tests, expressing the null hypothesis that the control group mean differential activation was zero (e.g., spatial delay activity equaled verbal delay activity); (2) two-sample unpaired $t$ tests, expressing the null hypothesis that the difference between the control group and a patient was zero for a given contrast between task conditions; and (3) we split the control subjects into high and low DART groups, excluding the median subject, and compared delay-related activations in high and low DART groups. When comparing a patient with the control group, we used the entire control group. We could not in practice study a large separate control population for each subject, and to subdivide our existing control group would entail too great a loss of statistical power. A single control group is acceptable because our patients were not outliers in terms of demographic details or DART estimates of premorbid intelligence, and the patients' behavioral data were, on average, equivalent to controls, for both spatial and verbal tasks even when adjusting for DART (with one exception; see Results).

For each contrast of interest, our standard statistical threshold was, for family-wise error (FWE) rate, $p<0.05$ corrected for whole-brain comparisons. For contrasts of instruction delay-related activity in the control group, we also applied the threshold FWE $p<0.05$ within reduced search volumes. This affords a more sensitive test in which previous or independent data can be used to define the region of interest (ROI). Our ROIs were defined as spheres with $8 \mathrm{~mm}$ radius centered on $\pm 34,44,32$ [area 46 ; the coordinate of local maximal differential activation for all tasks sets vs baseline in Sakai et al. (2006)]; $\pm 36,44,6$ [area 10; the left-hemisphere correspondence from the rostral local maximal differential activation for task set vs baseline in Sakai et al. (2003)]; $\pm 30,56,6$ [area 10 from Sakai et al. (2006)]; and -54, 04, 22 (area 44; the local maximum for the verbal instruction delay-related activity in control subjects).

For contrasts expressing the interaction between task-related activation and group, we first applied the threshold with FWE $p<0.05$ corrected for whole-brain comparisons (e.g., was verbal instruction delay activity vs baseline different between a patient and the control group at any brain location with FWE $p<0.05)$. We then applied a threshold with false discovery rate (FDR) $p<0.05$. We then set an uncorrected threshold $p<0.05$ with secondary correction within a reduced search volumes. For letter instruction delays, the reduced search volume was a sphere of $8 \mathrm{~mm}$ radius centered on $-54,04,22$ (area 44; at the local maximum for the verbal instruction delay-related activity in control subjects). For the spatial instruction delay, we used two spheres of interest centered on $-24,-8,56$ (the SFS peak in control subjects for spatial instruction delay) and $-30,-52,56$ (the parietal local maximum for spatial instruction delay-related activity in controls). For both verbal and spatial instruction delays, we used ROI spheres of $8 \mathrm{~mm}$ radius centered again on the prefrontal peaks of task-set activation from Sakai et al. $(2003,2006)$ at $\pm 36,44,6 ; \pm 34,44$, 32 ; and $\pm 30,56,6$.

Time series were extracted from spherical ROIs of $5 \mathrm{~mm}$ radius centered on the presupplementary motor area (pre-SMA; -10, 10, 50) (Picard and Strick, 1996), Broca's area (area 44: $-54,04,22$ ), the fusiform word form area $(-36,-64,-14)$, area $8(-24,-8,56)$, parietal cortex $(-30,-52,56)$ for each subject. These peaks were identified from the second-level analyses of task-related activations during the instruction delay (vs baseline). The pre-SMA was chosen because it was a potential contributor to top-down control of caudal set-related activations. It was intact in all patients and was active during instruction delay for both modalities (see Fig. $4 A$ ) but did not show differential activation. The other areas showed differential activation according to modality but had not shown a reduction in activation in the patients.

Time series data were adjusted for task effects (removing drifts and scans with spikes or significant movements) for each subject individually and segregated for periods of instruction delay for each modality (from $6 \mathrm{~s}$ after onset of instruction delay to $6 \mathrm{~s}$ after the first stimulus presentation reflecting the delay to peak hemodynamic response). The correlations between Broca's area and the fusiform gyrus, the pre-SMA and the fusiform cortex, area 8 and parietal cortex, and the pre-SMA and area 8 were calculated for each subject and entered into a between-groups, fourway repeated-measures ANOVA, with group (control vs patient), connection (four connections), switch versus stay, and modality (verbal vs spatial) as factors. Statistical inferences ( $p$ values) are reported after Greenhouse-Geisser correction for nonsphericity.

\section{Results}

\section{Subject details}

Four patients completed the study and were comparable to the control group in age, sex, and DART scores (Table 1). Their lesions are shown in Figure 2 superimposed on the T1 canonical brain in normal space. The cross-hairs indicate the location of "all tasks" set-related activity from Sakai et al. (2006), and the center of the ROI was used for small-volume correction. All lesions affected this region (white color in Fig. 2).

\section{Behavioral data}

The reaction times and accuracies for the spatial and verbal tasks are shown for the control group and four patients in Figure 3, A and $B$, respectively. The accuracy of responses was lower for switch trials than stay trials $\left(F_{(1,21)}=14,1 ; p<0.001\right)$, but there was an interaction with group as shown in Figure $3 C\left(F_{(1,21)}=\right.$ 12.6; $p<0.005)$, with patients making more errors on switch trials and fewer on stay trials (within-patient difference between switch and stay: $t=3.7 ; p<0.01$ ), whereas control subjects' differences were minimal (Fig. $3 C$ ). There was no main effect of task modality $\left(F_{(1,21)}=3.0\right.$; NS) or interaction between group and modality $\left(F_{(1,21)}=1.8 ; \mathrm{df}=1,21 ; \mathrm{NS}\right)$. There was no third- 

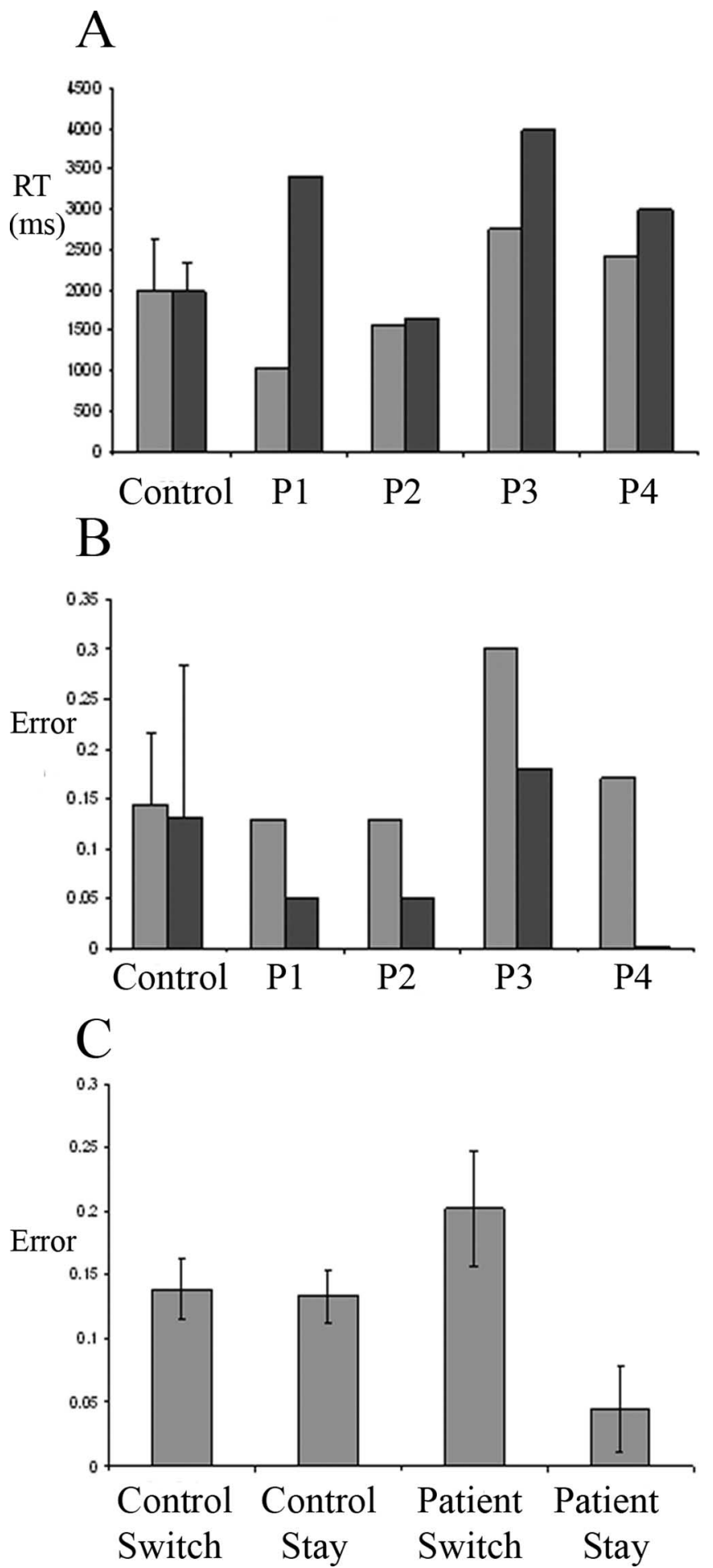

Figure 3. $\quad \boldsymbol{A}$, Reaction times (RT). $\boldsymbol{B}$, Error rates for the control group and each patient (P1P4). In $\boldsymbol{A}$ and $\boldsymbol{B}$, spatial tasks are indicated by light gray bars, and verbal tasks are indicated by dark gray bars. $\boldsymbol{C}$, Error rates are shown separately for trials in which the modality had just switched (switch) or was the same as the previous trial (stay), for control and patients groups, respectively, averaging across modality. Control data are shown as group mean $\pm \mathrm{SE}$.

order interaction $\left(F_{(1,21)}=0.7\right.$; NS) or main effect of group $\left(F_{(1,21)}=0.1\right.$; NS). In view of high variance in the verbal switch errors for patients, we performed a second restricted ANOVA of switch versus stay for the spatial task, with group as the betweensubject factor. There was again an effect of switch $\left(F_{(1,21)}=5.5\right.$; $p<0.03)$ with an interaction with group $\left(F_{(1,21)}=9 ; p<0.005\right)$, but no main effect of group $\left(F_{(1,21)}=0.8 ; \mathrm{NS}\right)$.
The reaction times did not differ between switch and stay trials $\left(F_{(1,21)}=2.6\right.$; NS), nor was there an interaction between switching and group $\left(F_{(1,21)}=0.2 ; \mathrm{NS}\right)$. There was a trend toward longer reaction times in patients $\left(F_{(1,21)}=3.3 ; p<0.10\right)$. In contrast to the results for accuracy, there was a main effect of modality $\left(F_{(1,21)}=8.8 ; p<0.01\right)$, although this was primarily driven by the interaction between modality and group $\left(F_{(1,21)}=\right.$ 9.4; $p<0.01$ ), with patients taking longer for verbal trials as shown in Figure 3A. There was no interaction between modality and switching $\left(F_{(1,21)}=0.7\right.$; NS $)$ and no higher-order interaction between modality, switching, and group $\left(F_{(1,21)}=2.6\right.$; NS).

In control subjects, the verbal reaction times correlated with DART $\left(r^{2}=0.28 ; \mathrm{df}=1,18 ; p<0.05\right)$. Three patients $(1,2$, and 4) had mean reaction times within the $95 \%$ confidence intervals adjusted for DART. In control subjects, the spatial-task reaction time did not correlate with DART $\left(r^{2}=0.10 ; \mathrm{df}=1,18 ; p=\mathrm{NS}\right)$, and no patient lay beyond $95 \%$ confidence limits. Verbal accuracy in controls did correlate with DART $\left(r^{2}=0.35\right.$; $\mathrm{df}=1,18$; $p<0.01$ ), but no patient lay beyond $95 \%$ confidence limits for accuracy when adjusted for DART. Spatial accuracy did not correlate with DART in controls $\left(r^{2}=0.06 ; \mathrm{df}=1,18 ; p=\mathrm{NS}\right)$, and no patient lay beyond $95 \%$ confidence limits.

\section{Imaging data: control subjects}

Instruction delay

Figure $4 A$ shows spatial instruction delay-related activity versus baseline (green) and verbal instruction delay-related activity versus baseline (red) for the control participants (details in supplemental Table S1, available at www.jneurosci.org as supplemental material). At a standard threshold (FWE, $p<0.05$ ) there was no prefrontal activation associated with instruction delay. However, for letter instruction delay, there was significant activation (vs baseline) when corrected for a reduced search volume in the area 10 ROI centered on $-36,44,06$ (cf. Sakai et al., 2003) at $-36,36$, $6(t=3.51 ; p<0.01)$ and $-38,38,10(t=3.51 ; p<0.01)$. There were no corresponding peaks of significant activation (vs baseline) on the right hemisphere or in area 46 ROIs centered on \pm 34 , 44,32 or area 10 ROIs centered or $\pm 30,56,6$.

For spatial instruction delay, there were peaks of activation (vs baseline) in the left area 46 ROI centered on $-34,44,32$ at -36 , $38,32(t=3.38 ; p<0.05)$ but not on the right area nor in the area 10 ROIs centered on $\pm 36,44,6$ or $\pm 30,56,6$. This region of significant spatial instruction delay-related activity in control subjects was included in the lesions in all subjects.

In Figure $4 A$, an impression is gained that the ventral regions are more active when anticipating a verbal working memory task, and dorsal regions more active when anticipating a spatial working memory task. This was tested formally with contrasts of letter versus spatial instruction delay activity and vice versa (Fig. $4 B$ ). Letter instruction delay activation was greater than spatial instruction delay activation in Broca's area 44 (when thresholded within the reduced search volume) with the peak difference at $-60,2,18(t=3.58 ; p=0.004)$. Spatial instruction delay activation was greater than letter instruction delay activation in the frontal eye fields (area 8; $-24,-8,56 ; t=5.47 ; p=0.005$, thresholded at initial FWE 0.05). Additional specific activation in the spatial instruction delay (vs verbal instruction delay) was seen with a more liberal threshold FDR correction of $p<0.05$ at 26, $-10,52(t=4.59 ; p=0.019) ; 26,4,56(t=4.48 ; p=0.023) ; 10$, $-58,54(t=4.52 ; p=0.021) ; 44,-38,44(t=4.52 ; p=0.021)$; $-12,-60,56(t=3.97 ; p=0.031)$; and 50, $8,20(t=3.93 ; p=$ $0.033)$. This is shown in Figure $4 B$, using an equivalent global threshold of $p<0.001$ uncorrected for illustrative purposes. 


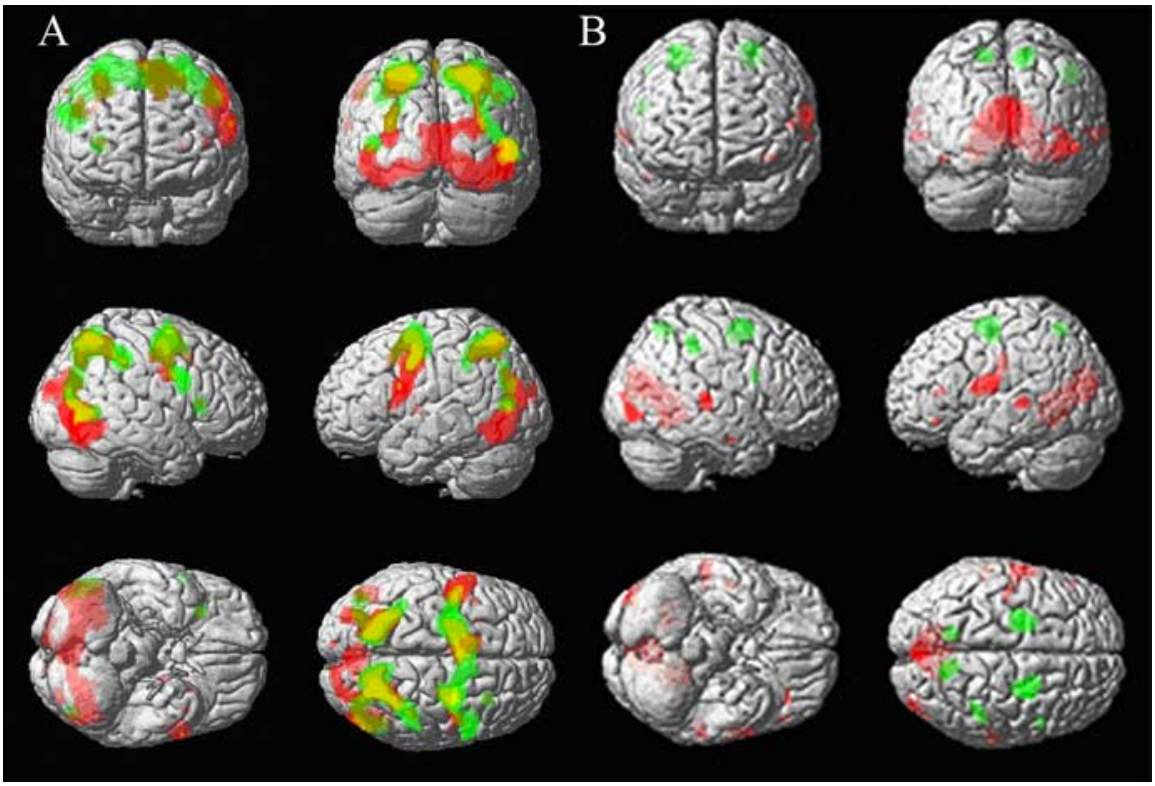

Figure 4. SPM $\{t\}$ maps of activations during the instruction delay, before subjects have seen the stimuli that are to be remembered. $\boldsymbol{A}$, Thresholded at $p<0.05$ (FWE) for verbal (red) and spatial (green) instruction delays against baseline. $\boldsymbol{B}$, Thresholded at $p<0.001$ (uncorrected) for the difference between modalities. Activations that were greater for verbal than spatial instruction delays are shown in red, whereas activations that were greater for spatial than verbal instruction delays are shown in green. See Results for corrected significance levels within ROIs. SPM\{t\}s are shown superimposed on a canonical T1-weighted image in MNI space.

We had predicted rostral prefrontal activation during both instruction delays. Conjoint activation was tested using the "conjunction null" hypothesis, requiring rejection of the null hypothesis for both verbal and spatial instruction delay-related activations. There were no prefrontal regions with significant conjoint activation for both letter and spatial delays (vs baseline) identified within any of the three left or right prefrontal ROIs.

\section{Memory delay}

Letter memory delay activity did not exceed spatial memory delay activity at global FWE 0.05 but did differ within the Broca's area 44 reduced search volume, with peak difference at $-58,2,16(t=$ $4.02 ; p=0.014)$. Spatial memory delay activity exceeded letter memory delay activity at a number of foci including the medial parietal cortex, intraparietal cortex, lateral occipital cortex, and superior frontal sulci bilaterally. The latter regions of superior frontal sulci lie within the spatial working memory region identified by Courtney et al. (1998). Full details are given in supplemental Table S2 (available at www.jneurosci.org as supplemental material). Conjoint letter and spatial memory delay activity (vs baseline) was found within the prefrontal reduced search volumes with peak at $-38,42,28(t=3.33 ; p=0.013) ;-28,46,10$ $(t=3.61 ; p<0.01) ;-28,50,10(t=3.02 ; p<0.05)$; and 32, 50, $10(t=3.14 ; p<0.05)$.

Letter instruction delay activity (vs baseline) did not differ between high and low DART subjects either at global FWE 0.05, global FDR 0.05 , or within the prefrontal reduced search volume $(-32,44,32 ; 8 \mathrm{~mm}$ radius). However, it did differ within the reduced search volume centered on Broca's area $44(-54,04,22$; $8 \mathrm{~mm}$ radius $)$ with a peak difference at $-56,6,22(t=3.31 ; p=$ $0.038)$. There were no voxels with a significant difference in spatial instruction delay-related activity (vs baseline) between high and low DART groups, at FWE 0.05 or FDR 0.05 , or in the prefrontal reduced search volume.
Imaging data for patients: each patient versus controls

Patient 1. For the letter instruction delay (vs baseline), there was no reduction or increase in activation at thresholds FWE 0.05 or FDR 0.05 and none in reduced search volumes. For the spatial instruction delay, there was no reduction or increase in activation at thresholds FWE 0.05 or FDR 0.05 and none in the reduced search volumes.

Patient 2. For the letter instruction delay (vs baseline), there was no reduction or increase in activation at thresholds FWE 0.05 or FDR 0.05 and none in reduced search volumes. For the spatial instruction delay, there was no reduction or increase in activation at thresholds FWE 0.05 or FDR 0.05 and none in the reduced search volumes.

Patient 3. For the letter instruction delay (vs baseline), there was no reduction or increase in activation at thresholds FWE 0.05 or FDR 0.05 and none in reduced search volumes. For the spatial instruction delay, there was one focus of reduced activation at FWE 0.05 anterior to the lesion (at $-14,44,30 ; t=8.67 ; p=0.003$ ) but no additional difference at FDR 0.05 and none in reduced search volumes. This anterior focus was not associated with significant spatial instruction delay activity in controls. There were no areas of increased activation, at FWE, FDR, or in the reduced search volumes.

Patient 4. For the letter instruction delay (vs baseline), there was no reduction in activation at FWE 0.05, none at FDR 0.05, and none in the reduced search volumes; however, there was increased activation at $-34,8,-10(t=7.20 ; p=0.02$, FWE correction). For the spatial instruction delay, there was none at FWE 0.05, none at FDR 0.05, and none in reduced search volumes. However, there were regions of increased activation (vs baseline) in the right motor cortex at 38, $-26,60$ (FWE, $p<0.05$ threshold); in the left motor cortex at $-26,-28,76$; in the preSMA at 8, 8, 60; in the anterior cingulate at $0,40,18$; and in the posterior cingulate cortex at $0,-32,44$ (FDR, $p<0.05$ threshold). There was no significant increase in activation of the contralateral prefrontal cortex even within the reduced search volumes.

The magnitude activity for spatial and verbal instruction delays is plotted for area 8 and area 44, respectively, in Figure 5. These plots rank the entire study population, indicating the instruction delay-related activation of patients relative to the control group.

\section{Imaging data: functional connectivity}

The correlations among the five regions (pre-SMA to frontal eye fields area 8 and area 44 , area 8 to parietal cortex, and area 44 to fusiform gyrus) were, overall, lower in the patients (main effect of group: $F_{(1,21)}=11 ; p<0.01$ ) and more so for stay trials (interaction between group and switch-stay: $\left.F_{(1,21)}=5.0 ; p<0.05\right)$ as shown in Figure $6 \mathrm{~A}$. The correlations differed between connections (main effect of connection: $F_{(1,21)}=14 ; p<0.001$ ) and between switch and stay trials (main effect of switch-stay: 


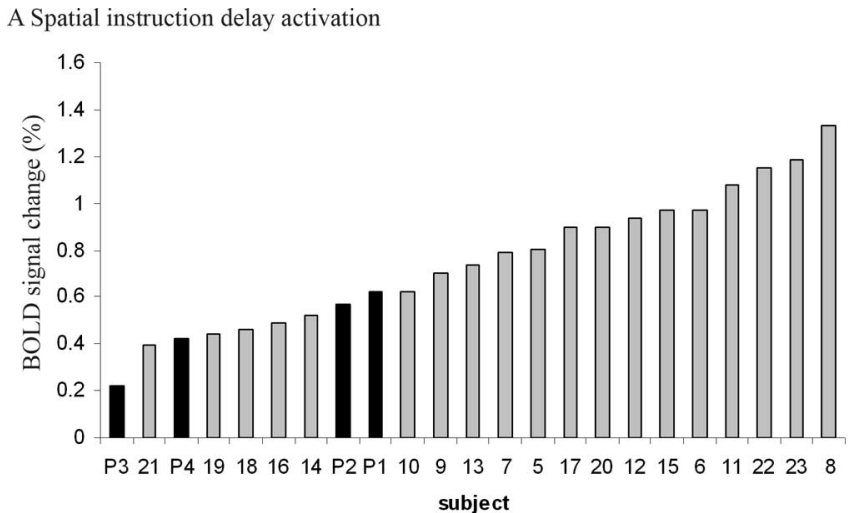

B Verbal instruction delay activation

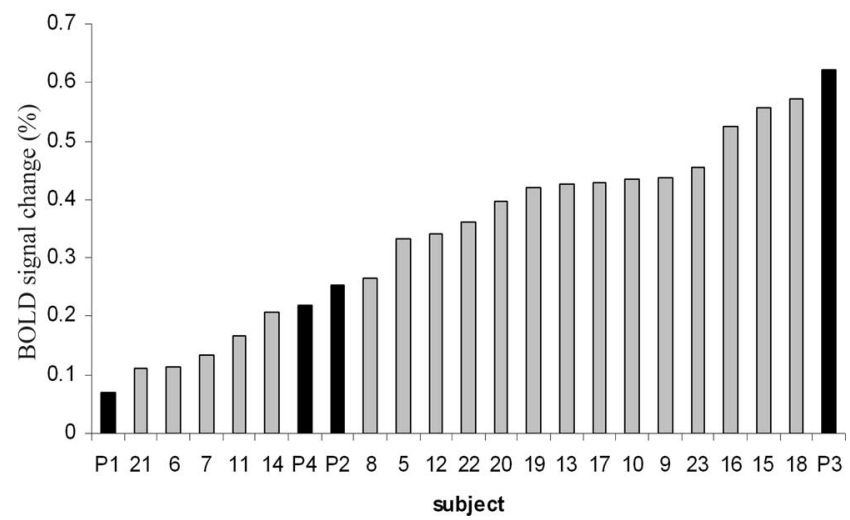

Figure 5. The set activity in area 8 at $-24,-8,56$ during spatial instruction delay (top) and in area 44 at $-54,04,22$ during verbal instruction delay (bottom) is plotted separately for each subject. The four patients (subjects P1-P4) are marked in black, and control subjects (subjects 5-23) are marked in gray. The instruction delay activity effect size is in arbitrary units indicating the BOLD signal change, derived from the contrast images of verbal instruction delay and spatia instruction delay, respectively.

$\left.F_{(1,21)}=5.7 ; p<0.05\right)$. There was no main effect of modality $\left(F_{(1,21)}=1.3 ; \mathrm{NS}\right)$.

There was an interaction between modality and the connection, indicating modality-specific changes in correlation during the instruction delay $\left(F_{(1,21)}=4.5 ; p<0.05\right)$ as shown in Figure $6 \mathrm{~B}$. Specifically, on stay trials, the connections of Broca's area (area 44) were greater during verbal instruction delays, whereas connections of the superior frontal sulcus were greater during spatial instruction delays. On switch trials, a more complex relationship emerges, with higher correlations of the pre-SMA on verbal trials, even in its connection with the superior frontal sulcus.

There were no interactions between connection and switchstay $\left(F_{(1,21)}=0.3\right.$; NS); modality and group $\left(F_{(1,21)}=0.2 ; \mathrm{NS}\right)$; connection and group $\left(F_{(1,21)}=2.2 ; \mathrm{NS}\right)$; connection and switch-stay $\left(F_{(1,21)}=0.3\right.$; NS); and modality and switch-stay $\left(F_{(1,21)}=0.3\right.$; NS $)$.

The interaction between modality and connection interacted with the switch-stay factor $\left(F_{(1,21)}=4.3 ; p<0.05\right)$. However, there was no interaction between the connection, switch-stay and group $\left(F_{(1,21)}=0.8\right.$; NS); connection, modality, and group $\left(F_{(1,21)}=2.8\right.$; NS); connection, switch-stay, and group $\left(F_{(1,21)}=\right.$ 0.8 ; NS); and modality, switch-stay, and group $\left(F_{(1,21)}=0.6\right.$; NS). There was no significant four-way interaction $\left(F_{(1,21)}=1.0\right.$; NS).
A

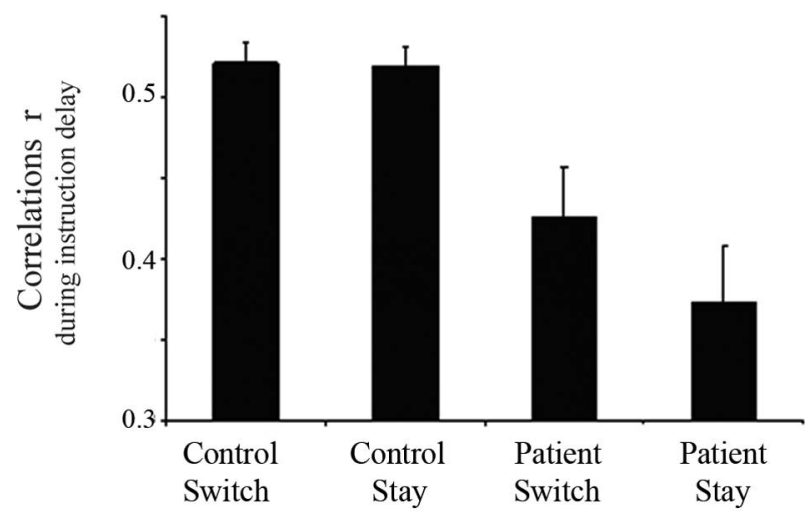

B

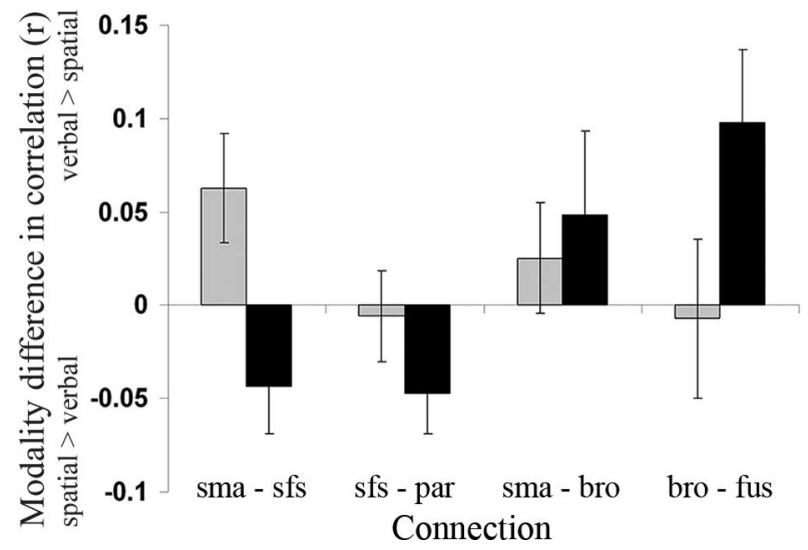

Figure 6. $\boldsymbol{A}$, The correlations $(r)$ among the pre-SMA, area 44, area 8 , and parietal and fusiform cortex were lower in patients, more so on stay trials (bars show group means averaged across all connections and both modalities $\pm \mathrm{SE}$ ). $\boldsymbol{B}$, The modality difference in correlation $r$, between spatial and verbal instruction delays, varied between the four anatomical connections and depended on the switch-stay factor but not the group (see Results for ANOVA results). A positive value indicates higher correlations during verbal instruction periods, whereas a negative value indicates higher correlations during spatial instruction periods. Black bars represent stay trials, and gray bars represent switch trials. sma, Pre-SMA; sfs, superior frontal sulcus; par, parietal cortex; bro, area 44 of Broca's area; fus, fusiform gyrus.

\section{Discussion}

The aim of the present experiment was to test whether the rostral prefrontal cortex is essential for setting up preparatory activity in posterior areas that are involved in processing the specific task items. We were able to record such posterior set activity in the control participants during the instruction delay. It is clear that the activity is prospective. It is found in spatial areas if the task is to remember locations and in verbal and visual areas if the task is to remember letters (Fig. $4 B$ ). Thus, the activity does not reflect memory for the words of the instruction but rather preparation for processing in a particular domain. In addition, this preparation was associated with increased coupling between areas related to the modality of the anticipated task.

If rostral regions of the left prefrontal cortex are essential for setting up prospective activity in task-related areas, we would have expected to find a decrease in the set activity in these areas in the patients. Instead, we found normal set activity within each region during the instruction delays in each patient. The apparent absence of reduced regional activations was despite liberal thresholding for effects within a reduced search volume equivalent to just one-half resel. Moreover, because patient 3 had the highest activity of all subjects in area 44 during instruction delay, our failure to detect reduced activation here is clearly not just a type II error. Statistical power may have been limited to detect 
differences in other regions or other patients. However, we had a similar number of trials of each type as used by Sakai and Passingham (2002) in previous work and may have gained power using 3 Tesla MRI.

There are three alternative explanations for the normal task set activity in patients. The first is that lesions were chronic. Although one patient had surgery only 8 months before testing, even his lesion is likely to have existed for months if not years beforehand. This would have given time for plasticity and the transfer of normal functions to adjacent or contralateral prefrontal cortex that remained intact. It could be that acute lesions from stroke or trauma would have resulted in less set activity.

The second reason is that our lesions were unilateral. The intact right prefrontal cortex might be able compensate for the loss of tissue in the left hemisphere. We found no evidence for compensatory overactivation in the right prefrontal cortex. But it still remains that the right rostral prefrontal cortex may have been sufficient to establish task set activity.

A final reason is that our lesions were in the left hemisphere. Studies based on functional neuroimaging often emphasize laterality effects in a variety of cognitive processes, including working memory, encoding, and retrieval. However, few studies have formally assessed laterality effects, relying instead on differences in thresholded images. For example, rule-set maintenance has been associated with right anterior prefrontal activations (Sakai and Passingham, 2003), left homologous activations (Sakai and Passingham, 2006), or bilateral activations (Bunge et al., 2003) at standard thresholds. However, we cannot exclude the possibility that right rostral prefrontal lesions would have had a greater effect.

Although the patients had normal set activity, they did not have a normal pattern of brain connectivity (Fig. 6). This confirms the suggestion made by Sakai et al. (2002) that the prefrontal cortex modulates the strength of connections between posterior areas. They found that the correlation between activity in areas 8 and 7 was dependent on the activation in prefrontal area 46. Moreover, they found that on a spatial delayed response task, the covariance between the delay-related activity in areas 8 and 7 was related to errors, whereas the magnitude of activity was not. The importance of abnormal connectivity in the presence of normal regional activations has been shown in other patient populations in the language and motor systems: fronto-temporal connectivity correlates with primary progressive aphasia in a language paradigm, whereas activation within the frontal and temporal areas does not (Sonty et al., 2007); and in Parkinson's disease, the SMA can be functionally disconnected from prefrontal afferents, without focal underactivation (Rowe et al., 2002). Together, these data suggest that the functional connectivity is at least as important as the magnitude of regional activations for accurate performance and may be more sensitive to cerebral pathology. Our data show additionally that functional connectivity is impaired even among intact brain regions, after lesions of the rostral prefrontal cortex.

What, then, is the significance of this reduced connectivity? Our data should be interpreted in the light of what we know about delay-related activity on delayed response tasks. These require the subject, whether monkey or human, to hold in mind for the current trial the intention to respond to a particular location. During the delay period, there is sustained activity in neurons in areas 46, 8, and 7 (Fuster, 1973; Goldman-Rakic, 1987; Chafee and Goldman-Rakic, 1998; Sawaguchi and Yamane, 1999). However, disruption of that activity in either area 8 (Goldman and Rosvold, 1970) or parietal area 7 (Chafee and Goldman-Rakic,
2000) does not cause an impairment, whereas selective lesions or inactivation of prefrontal area 46 produces a severe impairment (Butters et al., 1971; Sawaguchi and Iba, 2001). Our data suggest that impaired functional connectivity among posterior taskrelated brain regions, resulting from lesions of area 10 or 46, may mediate this poor performance.

Like delayed response tasks, the present task requires that subjects keep in mind the specific intention for the current trial. In healthy humans, such intentions are associated with activation of rostral prefrontal cortex including area 10 (Haynes et al., 2007) and area 46 (Lau et al., 2004). Lesion studies have also implicated these areas in "prospective memory," which is the holding of a delayed intention for a secondary task (Burgess et al., 2000) and the general ability to use advanced information to guide future responses (Drewe, 1974; Nelson, 1976; Karnath et al., 1991; Stablum et al., 1994; Rogers and Monsell, 1995; Johns, 1996; Koski et al., 1998; Rogers et al., 1998; Lepage and Richer, 2000; Warrington, 2000; Gomez-Beldarrain et al., 2004). Because our subjects knew that the task modalities would change at some point, they may also have maintained the prospective memory that a switch will later be required. Such meta-cognitive processes govern intentions that span several subordinate tasks and are associated with frontal polar cortex (Koechlin et al., 1999), but they are not restricted to within trials and would not thus appear in our contrast images.

In the present study, the patients had impaired functional connectivity in both the spatial and verbal networks and on both stay and switch trials. However, the reduction was greater on stay trials. Conversely, our patients made more errors than the control participants on switch trials. Switch deficits after frontal lesions have been interpreted as a loss of a specialist "switch" function within prefrontal cortex (Owen et al., 1993; Dias et al., 1997; Manes et al., 2002; Swainson et al., 2003; Aron et al., 2004; Mayr et al., 2006). However, our patients also made fewer errors than the control participants on stay trials, despite the reduced connectivity. This suggests an alternative interpretation. The default strategy is to process the items in the same way as on the previous trial. On switch trials, it is only by holding the current intention in mind that one can countermand this default. We therefore suggest that the patients were impaired in holding the current rule in mind and cite the reduced errors on stay trials as evidence for a preference for the default strategy. The same interpretation can account for the fact that it is on switch trials and not stay trials that monkeys with prefrontal lesions make errors on delayed response (Diamond and Goldman-Rakic, 1989).

Our interpretation would have been strengthened if we could have compared prefrontal activity on error trials with activity on correct trials. Unfortunately, we could not do this as a result of lack of power because of insufficient error trials. We predict that even in healthy participants, there would be reduced activation in areas 10 and 46 on error trials compared with correct trials. The basis for this prediction is the pattern of results in a previous paper on error trials on a spatial memory task (Sakai et al., 2002). It would also have been helpful to see whether there were differences in regional activations between switch and stay trials for each modality in patients compared with controls. This could be looked at in future studies with more trials per subject, collected perhaps over several scanning sessions. We predict that in patients the covariance among posterior regions would be especially reduced ahead of those switch trials on which they made errors.

We conclude that the prefrontal cortex is essential for holding the current rule and thus the current intention in mind. Activity in prefrontal cortex is needed for maintaining the normal corre- 
lations between the task-related areas. To put this more generally, when we interpret the effects of a lesion, we need to take into account that it may disturb the normal interactions between those areas that remain intact.

\section{References}

Aron AR, Monsell S, Sahakian BJ, Robbins TW (2004) A componential analysis of task-switching deficits associated with lesions of left and right frontal cortex. Brain 127:1561-1573.

Blair J, Spreen O (1989) Predicting premorbid IQ: a revision of the National Adult Reading Test. Clin Neuropsychologist 3:129-136.

Bunge SA, Kahn I, Wallis JD, Miller EK, Wagner AD (2003) Neural circuits subserving the retrieval and maintenance of abstract rules. J Neurophysiol 90:3419-3428.

Burgess PW, Veitch E, de Lacy Costello A, Shallice T (2000) The cognitive and neuroanatomical correlates of multitasking. Neuropsychologia 38:848-863.

Butters N, Pandya D, Sanders K, Dye P (1971) Behavioral deficits in monkeys after selective lesions within the middle third of sulcus principalis. J Comp Physiol Psychol 76:8-14.

Chafee MV, Goldman-Rakic PS (1998) Matching patterns of activity in primate prefrontal area $8 \mathrm{a}$ and parietal area 7ip neurons during a spatial working memory task. J Neurophysiol 79:2919-2940.

Chafee MV, Goldman-Rakic PS (2000) Inactivation of parietal and prefrontal cortex reveals interdependence of neural activity during memoryguided saccades. J Neurophysiol 83:1550-1566.

Courtney SM, Petit L, Maisog JM, Ungerleider LG, Haxby JV (1998) An area specialized for spatial working memory in human frontal cortex. Science 279:1347-1351.

D’Esposito M, Postle BR (1999) The dependence of span and delayed-response performance on prefrontal cortex. Neuropsychologia 37:1303-1315.

Diamond A, Goldman-Rakic PS (1989) Comparison of human infants and rhesus monkeys on Piaget's AB task: evidence for dependence on dorsolateral prefrontal cortex. Exp Brain Res 74:24-40.

Dias R, Robbins TW, Roberts AC (1997) Dissociable forms of inhibitory control within prefrontal cortex with an analog of the Wisconsin Card Sort Test: restriction to novel situations and independence from "online" processing. J Neurosci 17:9285-9297.

Drewe E (1974) The effect of type and area of brain lesion on Wisconsin Card Sorting Test performance. Cortex 10:159-170.

Friston KJ, Ashburner J, Poline J-B, Frith CD, Frackowiak RSJ (1995) Spatial registration and normalisation of images. Hum Brain Mapp 2:165-189.

Fuster JM (1973) Unit activity in prefrontal cortex during delayed-response performance: neuronal correlates of transient memory. J Neurophysiol 36:61-78.

Goldberg E, Podell K, Lovell M (1994) Lateralization of frontal lobe functions and cognitive novelty. J Neuropsychiatry Clin Neurosci 6:371-378.

Goldman PS, Rosvold HE (1970) Localization of function within the dorsolateral prefrontal cortex of the rhesus monkey. Exp Neurol 27:291-304.

Goldman-Rakic P (1987) Circuitry of primate prefrontal cortex and regulation of behaviour by representational memory. In: Handbook of physiology (Plum F, ed), pp 373-417. Bethesda, MD: American Physiological Society.

Gomez-Beldarrain M, Harries C, Garcia-Monco JC, Ballus E, Grafman J (2004) Patients with right frontal lesions are unable to assess and use advice to make predictive judgments. J Cogn Neurosci 16:74-89.

Haynes JD, Sakai K, Rees G, Gilbert S, Frith C, Passingham RE (2007) Reading hidden intentions in the human brain. Curr Biol 17:323-328.

Johns LC (1996) The effects of prefrontal damage in patients with surgical excisions, closed head injury, and gun-shot wounds. In: Linacre College, p 267. Oxford: Oxford UP.

Karnath HO, Wallesch CW, Zimmermann P (1991) Mental planning and anticipatory processes with acute and chronic frontal lobe lesions: a comparison of maze performance in routine and non-routine situations. Neuropsychologia 29:271-290.

Koechlin E, Basso G, Pietrini P, Panzer S, Grafman J (1999) The role of the anterior prefrontal cortex in human cognition. Nature 399:148-151.

Koski LM, Paus T, Petrides M (1998) Directed attention after unilateral frontal excisions in humans. Neuropsychologia 36:1363-1371.

Lau HC, Rogers RD, Haggard P, Passingham RE (2004) Attention to intention. Science 303:1208-1210.
Lepage M, Richer F (2000) Frontal brain lesions affect the use of advance information during response planning. Behav Neurosci 114:1034-1040.

MacDonald III AW, Cohen JD, Stenger VA, Carter CS (2000) Dissociating the role of the dorsolateral prefrontal and anterior cingulate cortex in cognitive control. Science 288:1835-1838.

Manes F, Sahakian B, Clark L, Rogers R, Antoun N, Aitken M, Robbins T (2002) Decision-making processes following damage to the prefrontal cortex. Brain 125:624-639.

Mayr U, Diedrichsen J, Ivry R, Keele SW (2006) Dissociating task-set selection from task-set inhibition in the prefrontal cortex. J Cogn Neurosci 18:14-21.

Miller EK, Cohen JD (2001) An integrative theory of prefrontal cortex function. Annu Rev Neurosci 24:167-202.

Nelson H (1976) A modified card sorting task sensitive to frontal lobe defects. Neuropsychologia 12:313-324.

Owen AM, Roberts AC, Hodges JR, Summers BA, Polkey CE, Robbins TW (1993) Contrasting mechanisms of impaired attentional set-shifting in patients with frontal lobe damage or Parkinson's disease. Brain 116:1159-1175.

Owen AM, Morris RG, Sahakian BJ, Polkey CE, Robbins TW (1996) Double dissociations of memory and executive functions in working memory tasks following frontal lobe excisions, temporal lobe excisions or amygdalo-hippocampectomy in man. Brain 119:1597-1615.

Picard N, Strick PL (1996) Motor areas of the medial wall: a review of their location and functional activation. Cereb Cortex 6:342-353.

Rogers R, Monsell S (1995) Costs of a predictable switch between simple cognitive tasks. J Exp Psychol 124:207-221.

Rogers R, Sahakian B, Hodges J, Polkey C, Kennard C, Robbins T (1998) Dissociating executive mechanisms of task control following frontal lobe damage and Parkinson's disease. Brain 121:815-842.

Rogers RD, Andrews TC, Grasby PM, Brooks DJ, Robbins TW (2000) Contrasting cortical and subcortical activations produced by attentional-set shifting and reversal learning in humans. J Cogn Neurosci 12:142-162.

Rorden C, Brett M (2000) Stereotaxic display of brain lesions. Behav Neurol $191-200$

Rowe J, Stephan KE, Friston K, Frackowiak R, Lees A, Passingham R (2002) Attention to action in Parkinson's disease: impaired effective connectivity among frontal cortical regions. Brain 125:276-289.

Rushworth MF, Passingham RE, Nobre AC (2002) Components of switching intentional set. J Cogn Neurosci 14:1139-1150.

Rushworth MF, Passingham RE, Nobre AC (2005) Components of attentional set-switching. Exp Psychol 52:83-98.

Sakai K, Passingham RE (2003) Prefrontal interactions reflect future task operations. Nat Neurosci 6:75-81.

Sakai K, Passingham RE (2006) Prefrontal set activity predicts rule-specific neural processing during subsequent cognitive performance. J Neurosci 26:1211-1218.

Sakai K, Rowe JB, Passingham RE (2002) Active maintenance in prefrontal area 46 creates distractor-resistant memory. Nat Neurosci 5:479-484.

Sawaguchi T, Iba M (2001) Prefrontal cortical representation of visuospatial working memory in monkeys examined by local inactivation with muscimol. J Neurophysiol 86:2041-2053.

Sawaguchi T, Yamane I (1999) Properties of delay-period neuronal activity in the monkey dorsolateral prefrontal cortex during a spatial delayed matching-to-sample task. J Neurophysiol 82:2070-2080.

Sonty SP, Mesulam MM, Weintraub S, Johnson NA, Parrish TB, Gitelman DR (2007) Altered effective connectivity within the language network in primary progressive aphasia. J Neurosci 27:1334-1345.

Spreen O, Strauss E (1991) A compendium of neuropsychological tests. New York: Oxford UP.

Stablum F, Leonardi G, Mazzoldi M, Umilta C, Morra S (1994) Attention and control deficits following closed head injury. Cortex 30:603-618.

Swainson R, Cunnington R, Jackson GM, Rorden C, Peters AM, Morris PG, Jackson SR (2003) Cognitive control mechanisms revealed by ERP and fMRI: evidence from repeated task-switching. J Cogn Neurosci 15:785-799.

Talairach J, Tournoux P (1988) A co-planar sterotactic atlas of the human brain. Stuttgart: Thieme.

Toni I, Schluter ND, Josephs O, Friston K, Passingham RE, Schluter N, Rushworth MF, Krams M, Turner R (1999) Signal-, set- and movementrelated activity in the human brain: an event-related fMRI study. Cereb Cortex 9:35-49.

Warrington EK (2000) Homophone meaning generation: a new test of verbal switching for the detection of frontal lobe dysfunction. J Int Neuropsychol Soc 6:643-648. 